\title{
Simulation of AH flows and deformation of DMD in a 3D AC
}

\author{
Zuhaila Ismail a, ${ }^{,}$, Lim Yeou Jiann ${ }^{\text {a, }}$ Sabaruddin Ahmad Jamali a, Alistair Fitt ${ }^{b}$ \\ a Department of Mathematical Sciences, Universiti Teknologi Malaysia, 81310 UTM Johor Bahru, Johor, Malaysia \\ ${ }^{b}$ Faculty of Technology, Design and Environment, Oxford Brookes University Headington Campus, Gipsy Lane, Oxford, OX3 OBP, United Kingdom \\ * Corresponding author: zuhaila@utm.my
}

Article history

Received 7 November 2017

Accepted 8 November 2017

\begin{abstract}
This paper presents the interaction between the aqueous humour $(\mathrm{AH})$ flows and the deformation of Descemet membrane detachment (DMD) in a $3 D$ anterior chamber (AC). Arbitrary Lagrangian Eulerian (ALE) method is used to model the problem. Finite element method using COMSOL Multiphysics software is adopted to solve the governing equations for the $\mathrm{AH}$ flows and the deformation of DMD. The fluid flow behaviour and the deformation of the detached Descemet membrane are analysed in order to comprehend the progression of the DMD in the AC due to the AH flows and vice versa. The re-attachment or re-detachment of the DMD is significantly affected by the $\mathrm{AH}$ flows. Advance treatment for the DMD can be developed based on a better understanding of the interaction between the $\mathrm{AH}$ flows and the DMD.
\end{abstract}

Keywords: Aqueous humour, descemet membrane detachment, arbitrary lagrangian eulerian

\section{INTRODUCTION}

Descement membrane detachment (DMD) is a human eye disease and it causes blindness. DMD happens in the region called as anterior chamber (AC). AC is bounded by cornea, iris and pupil and filled by aqueous humour $(\mathrm{AH})$ which has properties similar to water. Cornea is the clear dome like surface that exists at the front of a human eye and it covers the iris and the pupil, as shown in Figure 1. The cornea is made up by three main layers and two auxiliary layers, those are epithelium, stroma, endothelium, Browman layer and Descemet membrane (DM). Epithelium is the outermost layer and endothelium is the innermost layer of the cornea. Stroma is the intermedium layer. Moreover, Browman layer is the layer lies between the stroma and epithelium and DM is the layer lies between the stroma and endothelium. DMD happens when there is a tear or break on the DM which allows the AH flows into the subspaces between the stroma and $\mathrm{DM}$, and consequently separate the DM away from the stroma.

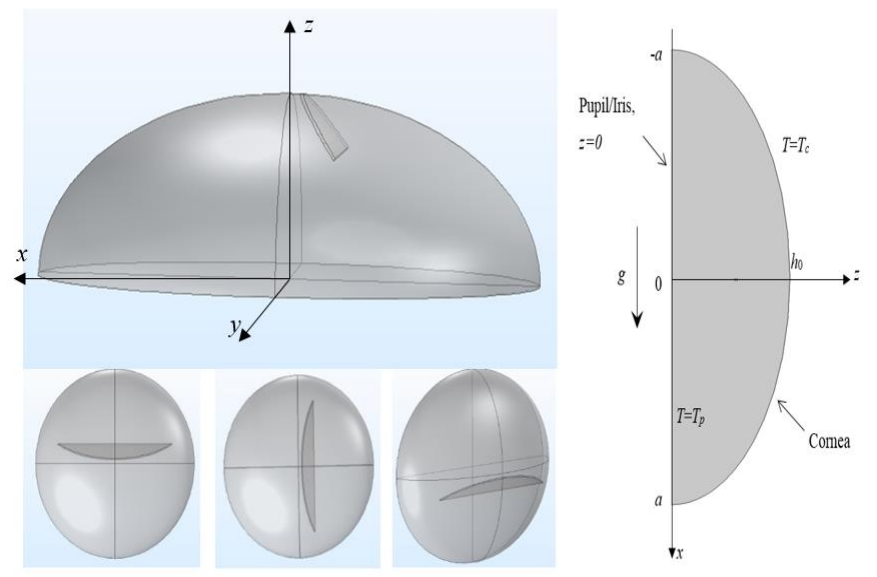

Fig. 1 Schematic diagram of the AC of human eye.
Mulhern et al. (1996), Potter and Zalatimo (2005) and Ünlü and Aksünger (2000) have reported that the DMD is induced by cataract surgery, iridectomy, trabeculectomy, corneal transplantation, deep lamellar keratoplasty, holmium laser sclerostomy, alkali burn and viscocanalostomy. The technique of curing of DMD caused by cataract surgery with sulphur hexafluoride injection is discussed by Sevillano et al. (2008). Further, 2005 studied the case of treating the DMD by injecting intracameral perfluoropropane $\left(\mathrm{C}_{3} \mathrm{~F}_{8}\right)$ into the $\mathrm{AC}$. Recently, Couch and Baratz (2009) investigated two cases of delayed bilateral DM, one eye was fixed surgically and the other eye improved spontaneously. They estimated that the spontaneous reattachment happen because of the $\mathrm{AH}$ flow in the $\mathrm{AC}$ driven by the buoyancy effects. Motivated by Couch and Baratz (2009), Ismail et al. (2013) developed a new mathematical model of the $\mathrm{AH}$ flow in the $\mathrm{AC}$ with DMD based on the work done by Canning et al. (2002), to explain the spontaneous reattachment phenomena. The authors concluded that the AH flow driven by the temperature difference across the eye and the orientation of the patient may control the clinical outcomes for the DMD.

Ismail et al. (2013) assumed that the AH flow in the AC is only driven by the gravity and buoyancy and hence, they considered the flow to be essentially two dimensional as explained in Canning et al. (2002). Therefore, they studied a two dimensional 'sectionally thinlayer' flow when a DMD is present in the AC. The surface of the cornea and the iris are assumed to be stationary tissue. The iris or pupil is assumed to have a fix temperature $T_{p}=302 \mathrm{~K}$. The cornea surface is imposed with a constant heat flux. The lubrication theory limit of the Navier-Stokes equations were employed by Ismail et al. (2013), in order to study the problem analytically. They concluded that the spontaneous reattachment of DMD is affected by the AH flow in AC.

However, the exact mechanisms of the spontaneous reattachment appears not to be known. This is because the interaction between the $\mathrm{AH}$ flows and the deformation of DMD is not considered in the previous studies. DMD in the flow stream of AH is subjected to fluid 
forces that will cause it to deform. The fluid forces are dependent on the geometry of the DMD and also the deformation, since the $\mathrm{AH}$ flows. Thus, the governing equations for the DMD as well as for the fluid need to be solved simultaneously. Lagrangian description is usually used to formulate the DMD, and Eulerian description is most often used to express the fluid for a convenient description of its convective nature. It is inconvenient to descript the fluid flow by using Lagrangian description which employs a referential frame moving with the flow to track the fluid motion. This is because a frequent remeshing is required due to large deformations due to the fluid motion, consequently increasing the required computational resources. On the other hand, if the Eulerian description is applied to descript the motion of DMD, the deformation of the DMD due to the fluid forces is difficult to track.

Therefore, the arbitrary Lagrangian Eulerian (ALE) technique is introduced which combine the advantage of both the methods and the obstacles of a purely Eulerian or purely Lagrangian implementation are avoided (Kjellgren and Hyva Ėrinen, 1998; Kuhl et al., 2003; Lo and Young, 2004; Braescu and George, 2007; Anjos et al., 2014; Jafari and Okutucu-Özyurt, 2016). The technique has been used by few researchers to investigate the human eye problem. The effect of intraocular pressure (IOP) variations $(10,20$, and $30 \mathrm{mmHg}$ ) in the stresses and deformations of the human eye components is analyzed by Karimi et al. (2016a) with the aid of a computational LagrangianEulerian coupling model. Karimi et al. (2016b) studied the stresses and deformations of all the human eye components due to the high explosive detonation by using fluid-structure interaction (FSI) model. Further, Karimi et al. (2016c) investigated the stresses and deformations of all the human eye components due to the tennis ball impact using a 3D computational dynamic fluid-structure interaction model.

The present study is aimed to deeply investigate the role of the AH flows in the deformation of the DMD using a 3D computational ALE model. The AH flows is modelled by using the fluid mechanical theory which developed by Canning et al. (2002) with the presence of detached DM. COMSOL Multiphysics which compute the results based on finite element method is applied to solve the model. Finite element methods is easily applied to objects with irregular geometry like the shape of the cornea. Moreover, it is convenient to solve problem with mixed boundary condition and problem with the objects composed of several different materials in its medium (for example, see Nouri et al., 2002; Marcon et al., 2002; Lewis et al., 2008; Reddy and Gartling, 2010; Zienkiewicz et al., 2013).

\section{MATHEMATICAL FORMULATION}

\section{Model Construction}

A three-dimensional AH flow driven by buoyancy effects in the $\mathrm{AC}$ with the presence of DMD has been considered. The temperature gradient is induced by the different temperature at the back of the $\mathrm{AC}$, which is estimated to core body temperature $\left(37^{0} \mathrm{C}\right)$ to the outside of the cornea (say $24^{0} \mathrm{C}$ ). Buoyant convection is happen within the $\mathrm{AC}$ due to the temperature gradient that occur across the $\mathrm{AC}$ of the eye. $z=0$ represent the plane formed by pupil aperture and the iris and the anterior surface of the cornea is represented by $z=h(x, y)=h_{0}(1-(x / a) 2-(y / a) 2) 1 / 2$, which the AH flow is introduced in a Cartesian coordinate $(x, y, z)$. At the iris, the temperature is fixed at $T_{p}$ which is close to the human body temperature, $310.15 \mathrm{~K}$, and the temperature at the cornea is assumed to be $T_{c}$, around $308.15 \mathrm{~K}$. The different temperatures between the back of the AC and the cornea induce the temperature gradient inside the AC. The patient is assumed to be in an upright position thus the gravity, $g$ is acting downward as shown in Figure 1. Following Canning et al. (2002) and Ismail et al. (2013), a set of typical values for the $\mathrm{AH}$ in the $\mathrm{AC}$ of the human eye is used as shown in Table 1.
Table 1: Properties of the $\mathrm{AH}$ used in the model.

\begin{tabular}{cll}
\hline Parameter & \multicolumn{1}{c}{ Description } & \multicolumn{1}{c}{ Unit } \\
\hline$a$ & Radius of the AC & $5.5 \times 10^{-3} \mathrm{~m}$ \\
$\alpha$ & $\begin{array}{l}\text { Coefficient of thermal } \\
\text { expansion }\end{array}$ & $3 \times 10^{-4} \mathrm{~K}^{-1}$ \\
& $\begin{array}{l}\text { Specific heat of the AH } \\
c_{P}\end{array}$ & $4200 \mathrm{Jkg}^{-1} \mathrm{~K}^{-1}$ \\
$g$ & Gravity & $9.8 \mathrm{~ms}^{-2}$ \\
$h_{0}$ & $\begin{array}{l}\text { Maximum height of the } \\
\text { cornea }\end{array}$ & $2.75 \times 10^{-3} \mathrm{~m}$ \\
$k$ & $\begin{array}{l}\text { Thermal conductivity of } \\
\text { the AH }\end{array}$ & $0.57 \mathrm{Wm}^{-1} \mathrm{~K}^{-1}$ \\
$\mu$ & Viscosity of the AH & $0.9 \times 10^{-3} \mathrm{kgm}^{-1} \mathrm{~s}^{-1}$ \\
$\rho$ & Density of the AH & $1000 \mathrm{kgm}^{-3}$ \\
$T_{C}$ & $\begin{array}{l}\text { Temperature at the } \\
\text { cornea }\end{array}$ & $308.15 \mathrm{~K}$ \\
$T_{p}$ & $\begin{array}{l}\text { Temperature at the } \\
\text { iris/pupil }\end{array}$ & $310.15 \mathrm{~K}$ \\
$U$ & Typical buoyancy-driven & $10^{-4} \mathrm{~ms}^{-1}$ \\
& flow speed in the AC & \\
\hline
\end{tabular}

The $\mathrm{AH}$ is assumed to be Newtonian, incompressible and driven by the temperature gradient. Therefore, the Navier-Stokes equations and energy equations which are derived based on the principle of conservation of energy are used to govern the fluid flow. The derivation of energy equations can be found in Batchelor (2000) and Kundu et al. (2012). Variation of density is happen due to the difference in temperature. The variation of the density cannot be neglected as it gives rise to the flow. This adds to the complexity in the analysis of the flow. Boussinesq approximation is applied to allow a more convenient procedure to obtain the solution. Boussinesq approximation Jaluria (1980) states that the density difference due to the interaction between the gravity is considered to vary with temperature where the density elsewhere is considered constant. Thus, the Boussinesq approximation governing equations of the $\mathrm{AH}$ flow driven by the temperature difference across the $\mathrm{AC}$ are:

$$
\begin{aligned}
& \frac{\partial u}{\partial x}+\frac{\partial v}{\partial y}+\frac{\partial w}{\partial z}=0, \\
& -\frac{\partial p}{\partial x}+v\left(\frac{\partial^{2} u}{\partial z^{2}}+\frac{\partial^{2} u}{\partial y^{2}}+\frac{\partial^{2} u}{\partial x^{2}}\right)-\rho\left(u \frac{\partial u}{\partial x}+v \frac{\partial u}{\partial x}+w \frac{\partial u}{\partial z}\right) \\
& +g\left(1-\alpha\left(T-T_{c}\right)\right)=0, \\
& -\frac{\partial p}{\partial y}+v\left(\frac{\partial^{2} v}{\partial z^{2}}+\frac{\partial^{2} v}{\partial y^{2}}+\frac{\partial^{2} v}{\partial x^{2}}\right)-\rho\left(u \frac{\partial v}{\partial x}+v \frac{\partial v}{\partial x}+w \frac{\partial v}{\partial z}\right)=0 \\
& -\frac{\partial p}{\partial z}+v\left(\frac{\partial^{2} w}{\partial z^{2}}+\frac{\partial^{2} w}{\partial y^{2}}+\frac{\partial^{2} w}{\partial x^{2}}\right)-\rho\left(u \frac{\partial w}{\partial x}+v \frac{\partial w}{\partial x}+w \frac{\partial w}{\partial z}\right)=0 \\
& \frac{k}{\rho C_{p}}\left(\frac{\partial^{2} T}{\partial x^{2}}+\frac{\partial^{2} T}{\partial y^{2}}+\frac{\partial^{2} T}{\partial z^{2}}\right)-u \frac{\partial T}{\partial x}+v \frac{\partial T}{\partial y}+w \frac{\partial T}{\partial z}=0
\end{aligned}
$$

where $v$ is the kinematic viscosity of the fluid. The boundary conditions for the velocity are:

$$
\begin{aligned}
& u(x, y, 0)=v(x, y, 0)=w(x, y, 0)=0, \\
& u(x, y, h(x, y))=v(x, y, h(x, y))=w(x, y, h(x, y))=0 .
\end{aligned}
$$

The boundary conditions for temperature are as follows:

$$
T=T_{p} \text { at } \mathrm{z}=0, T=T_{c} \text { at } z=h(x, y) .
$$

where $h_{0}$ is a typical depth of the AC, $T_{c}$ and $T_{p}$ denote the temperature at the cornea and the plane formed by the pupil and the iris respectively. By assuming that the fluxes and the pressures at each point $x$ are continuous, the pressure is known and is equal to the 
constant pressure $p=p_{a}$ at $x=a$, where $p_{a}$ is the normal atmospheric pressure and equal to $101325 \mathrm{~Pa}$. Finite element method is applied to solve equation (1) by subjected to equations (2) and (3).

Further, as a preliminary to the study, the detached DM is assumed to be a rigid, thin and small flap attached onto the anterior surface of the cornea and has the same temperature as the cornea, since originally it is a part of the cornea. Generally, DMD does not have a fix shape. For simplicity, in the present study, DMD is assumed to be a flat plate as shown in Figure 2. The Descemet's membrane is approximately $0.01 \mathrm{~mm}$ thick. The length of the DMD is taken to be $2 \mathrm{~mm}$. The cornea and the DMD are modelled as stationary tissues, so the posterior cornea surface and DMD surface are set to non-slip condition. The temperature of these surfaces is set to $308.15 \mathrm{~K}$. Also, the iris/pupil is modeled as stationary and the velocity is set to zero along the surface and temperature is set to $310.15 \mathrm{~K}$. Additionally, the problem domain and boundary conditions of the DMD are shown in Figure 2.
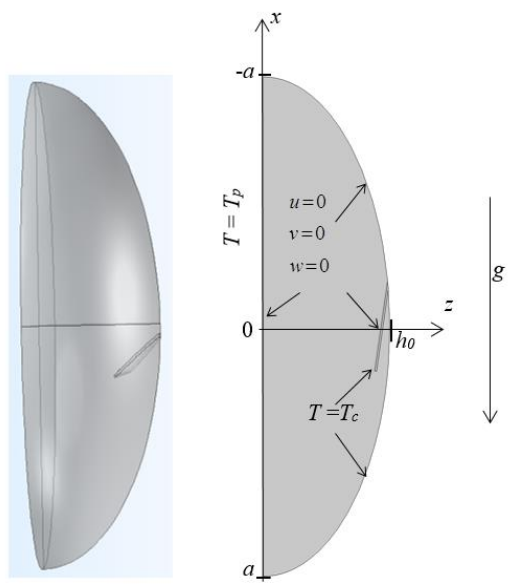

Fig. 2 Problem domain and boundary conditions of the DMD problem.

The computed approximation solutions of the fluid forces are then used to determine the deformation of the detach membrane. The DMD is assumed as a thin flexible elastic plate or beam. Krichhoff theory for small deflection of thin plate (see Ventsel and Krauthammer, 2001 and Ismail et al., 2013 for detail. ) is adopted to estimate the deformation of the DMD under the influence of the fluid flows. The detachment, $D(x, y, z)$ satisfies the following equation:

$$
D_{m}\left(\frac{d^{4} D}{d x^{4}}+\frac{d^{4} D}{d y^{4}}+\frac{d^{4} D}{d z^{4}}\right)=\nabla \cdot \sigma_{f},
$$

where $D_{m}$ is the flexural rigidity of the DMD and is defined as $D_{m}=E_{m} d_{m}^{3} / 12\left(1-v_{D}^{2}\right) . \quad E_{m}$ is the Young's modulus, $d_{m}$ is the thickness of the DMD and $v_{D}$ is the Poisson ration of the DM material. $\sigma_{f}$ is the fluid stress tensor which obtained from the previous calculation. Following Ismail et al. (2013), we set $E_{m}=50000 \mathrm{~Pa}^{d}, d_{m}=10 \mu \mathrm{mc}$ and $v_{D}=0.45$.

\section{Computational Mesh and Numerical Method}

COMSOL Multiphysics 5.2 was used to compute the numerical results. A personal computer with a processor speed of $2.30 \mathrm{GHz}$ and a RAM of $8 \mathrm{~GB}$ was used to perform the computation in present study. The governing equations (1) subjected to the boundary conditions (2) and (3) were first be solved by using finite element method. Then, the computed fluid forces were used to solve equation (4).

The three dimensional model is meshed using tetrahedral elements. The total number of elements in the mesh of the DMD problem (see Figure 3) is 33257 . In order to show that the results do not affected by the number of elements that used to mesh the geometry, a mesh test was conducted as shown in Figure $3 \mathrm{~b}$. The results did not depend on the number of element that use to mesh the geometry, thus, we hold the view that the results is independent from the number of elements. The desired variables such as velocity, pressure and temperature are approximated by using Lagrange cubic polynomial. The displacement of the DMD is also approximated by applying Lagrange cubic polynomial.

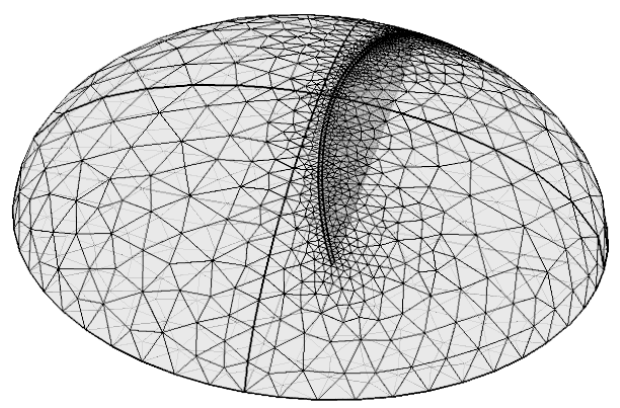

Fig. 3 Mesh plot.

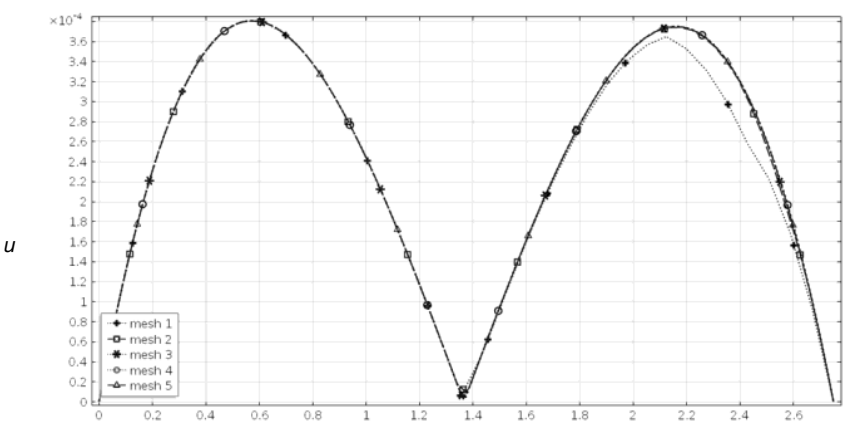

Fig. 4 Magnitude of the velocity along the line $(0,0,0)$ to $(0,0,0.0275)$ in the $A C$ without DMD with different mesh.

\section{RESULTS AND DISCUSSIONS}

Figure 4 shows the streamline plot for the fluid flow in the AC driven by the buoyancy convection without the DMD. The streamline is concur to the streamline plotted by Ismail et al. (2013) (see Figure 3 in Ismail et al., 2013). Moreover, the maximum flow speed observed in present communication is $3.680 \times 10^{-4} \mathrm{~m} / \mathrm{s}$ and it is located at position $\left(0,5.441 \times 10^{-4}\right)$. It is great agreement with the analytical determined maximum flow speed by Ismail et al. (2013) which is $3.962 \times 10^{-4} \mathrm{~m} / \mathrm{s}$ and it is located at $\left(0,5.811 \times 10^{-4}\right)$. This has validated the method applied in present study to compute the numerical solutions, as well as enhances our confidence to the results obtained in the research.

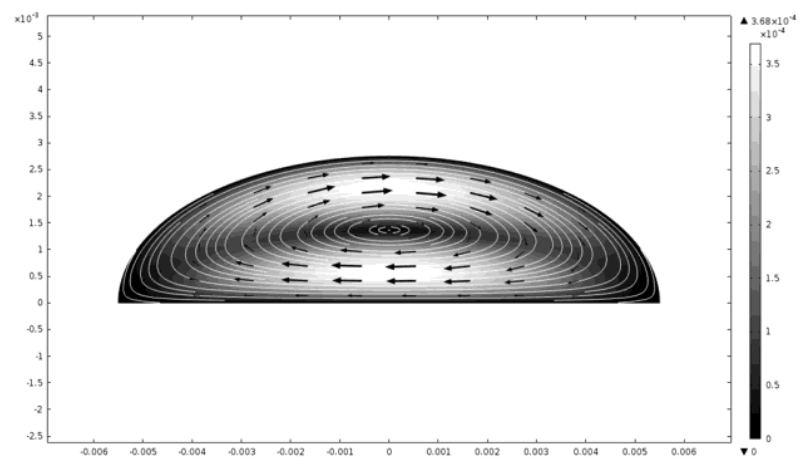

Fig. 5 Streamline plot at plane $y=0$ for case without DMD. 
The $\mathrm{AH}$ circulates in the $\mathrm{AC}$, as illustrate in Figures 5 for gravity acting upward in $x$-direction. As a result of the temperature gradient between the cornea and the iris/pupil, the $\mathrm{AH}$ adjacent to the iris/pupil is heated and eventually rises as buoyant flow. Then, the AH adjacent to the cornea becomes colder and hence becomes heavier than ambient fluid (see Figure 5). The existence of DMD in the AC yield an opposing effect on the buoyancy. The DMD increases the resistance to the $\mathrm{AH}$ flow, which is adjacent to the cornea, then reduces the velocity of the downward $\mathrm{AH}$ flow if compared with the case without DMD. In the subspace between the detached DMD and the stroma, the AH flow is able to turn around the corner and the flow is slow in this region and Moffatt vortices are detected Ismail et al. (2013) as illustrated in Figure 5. An opposite phenomena is observed when the gravity is assumed to act downward in the $x$-direction as shown in Figure 6.
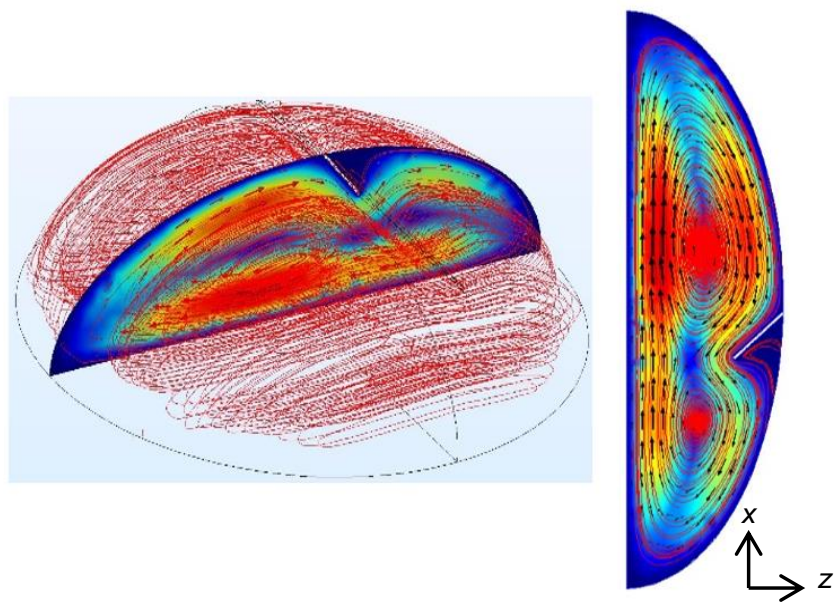

Fig. 6 Streamline in AC when the gravity is acting upward in $x$ direction.
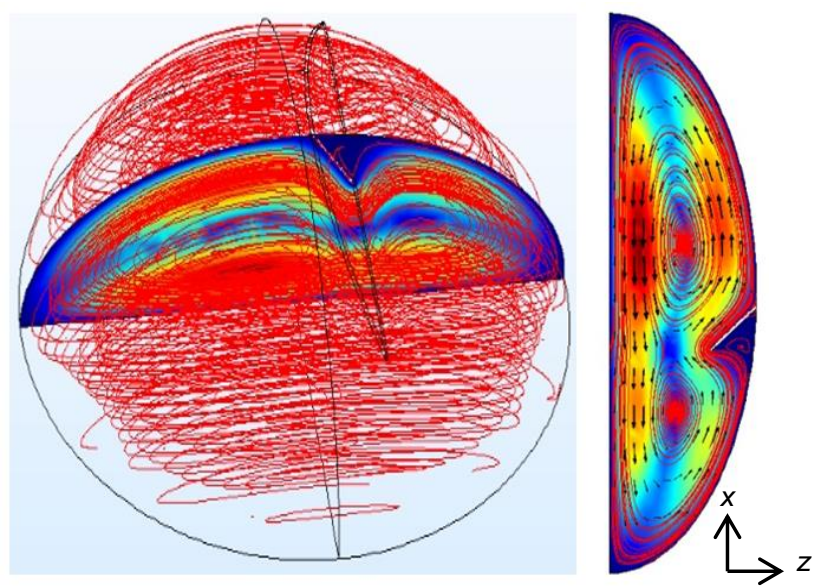

Fig. 7 Streamline in AC when the gravity is acting downward in $x$ direction.

The reattachment or redetachment of the DMD is contributed by the movement of the fluid in the subspace between the detached membrane and the stroma. As observed in Figure 7, the detached membrane is pushed away from its original position and toward to the stroma. This is because the pressure acting surrounding the DMD is larger than the pressure acting in the subspace between the detached membrane and the stroma, see Figure 8. This imply that the movement of the fluid surrounding the DMD is significant in process of reattachment or redetachment of the DMD. However,when the direction of the gravity is changed and in downward $x$-direction, the detached membrane is moved away from the stroma as shown in Figure 9. This is because the changing of the direction of the gravity has altered the $\mathrm{AH}$ flow in the $\mathrm{AC}$, thus the pressure distribution in the
$\mathrm{AC}$ is also altered as illustrated in Figure 10. The pressure in the subspace between the detached membrane and the stroma is higher than the pressure acting surrounding the DMD. Moreover, the flow of the $\mathrm{AH}$ in the $\mathrm{AC}$ also causes the DMD become more severe (see Figure 10).
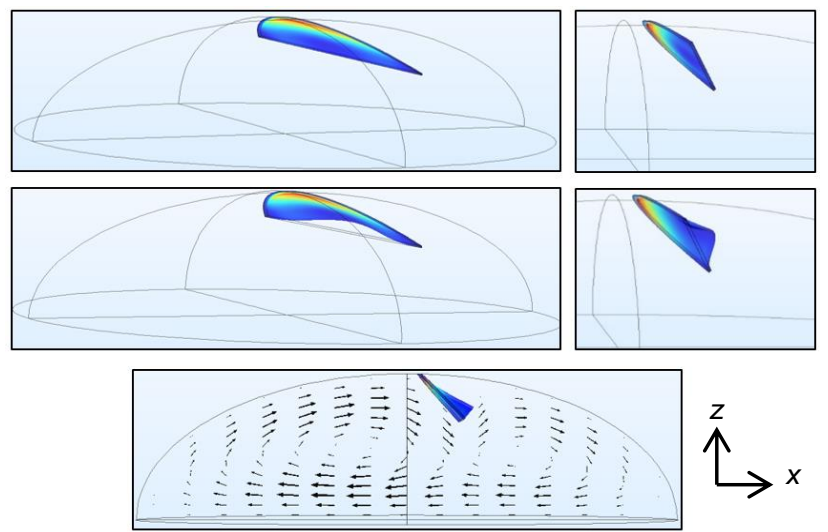

Fig. 8 Deformation of the DMD in AC when the gravity is acting upward in $x$-direction.

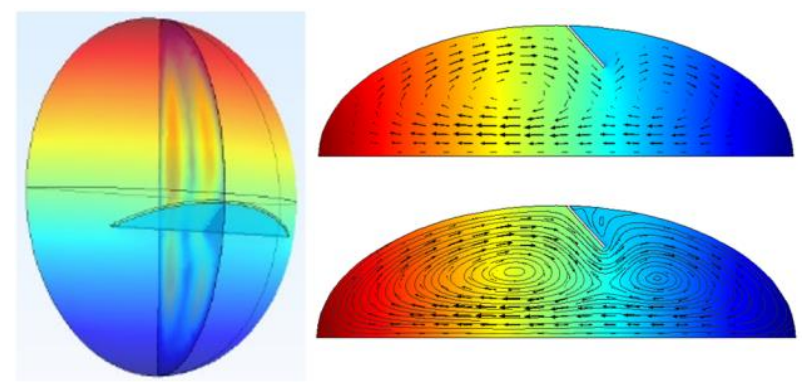

Fig. 9 .Pressure distribution in $\mathrm{AC}$ when the gravity is acting upward in $x$-direction.
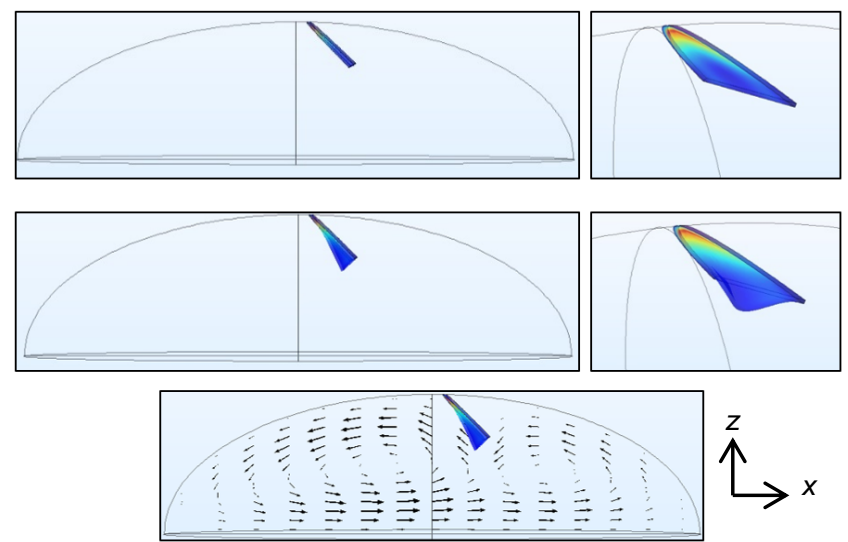

Fig. 10 Deformation of the DMD in AC when the gravity is acting downward in $x$-direction.

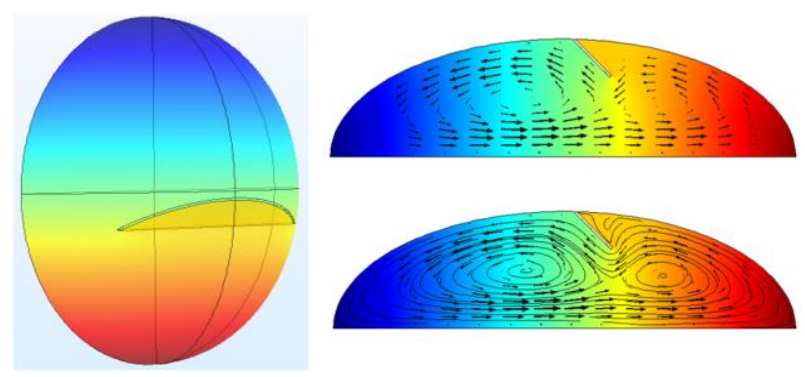

Fig. 11 Pressure distribution in $A C$ when the gravity is acting downward in $x$-direction. 
Therefore, we can conclude that the reattachment or redetachemnt of the DMD is depended on the $\mathrm{AH}$ flow, which driven by the temperature gradient, in the AC. We speculate that the reattachment of the DMD phenomena may only happen under certain conditions.

\section{CONCLUSIONS}

The behavior of the $\mathrm{AH}$ convection flow in a three dimensional $\mathrm{AC}$ in the presence of DMD have been numerically studied. The velocity streamline, pressure distribution and deformation of the DMD due to the fluid flow were obtained and illustrated graphically. Some interesting finding of the study can be summarized as follows:

1. The reattachment or redetachement of the DMD is depended on the $\mathrm{AH}$ flow which is driven by the temperature gradient between the iris/pupil and the cornea, in the AC .

2. The direction of the gravity has a significant effect to the $\mathrm{AH}$ flow in the AC. Therefore, the effect of the gravity need to be take into account in order to induce reattachment of the DMD.

3. The method proposed in present communication is appropriate and efficient in simulating the fluid flow in human eye as well as when different conditions are considered.

In our observation, to fully realize the behavior of the AC flow under the effect of the DMD, more research have to be done. For example, the fluid flow in the $\mathrm{AC}$ may be changed due to the blinking of the eye. This is because the temperature gradient in the $\mathrm{AC}$ is altered when the eye is blinking. In the future research, the blinking process of the human eye have to be considered in order to fully understand the dynamic of the $\mathrm{AH}$ flow in the AC with DMD. Further, the mechanisms of the reattachment or redetachment of the DMD is not completely developed. Many factors are not considered in present study such as the surface tension of the detached membrane, the rotation effect of the eye and so on.

\section{ACKNOWLEDGEMENT}

The authors would like to acknowledge KPT (MyBrain), MoHE and Research Management Centre-UTM for the financial support through vote numbers $03 \mathrm{G} 53$ and $13 \mathrm{H} 28$ for this research.

\section{REFERENCES}

Anjos, G. R., Borhani, I. N., Mangiavacchi, N., and Thome, J. R. (2014). A 3D moving mesh Finite Element Method for two-phase flows. Journal of Computational Physics 270:366-377.

Batchelor, G. K. (2000). An Introduction to Fluid Dynamics. New York: Cambridge University Press.

Braescu, L., and George, T. F. (2007). Arbitrary Lagrangian-Eulerian method for coupled Navier-Stokes and convection-diffusion equations with moving boundaries. 12th WSEAS International Conferences on Applied Mathematics. Location,

Canning, C. R., Greaney, M. J., Dewynne, J. N., and Fitt, A. D. (2002). Fluid flow in the anterior chamber of a human eye. IMA Journal of Mathematics Applied in Medicine and Biology. 19:31-60.

Couch, S. M., and Baratz, K. H. (2009). Delayed, Bilateral Descemet's Membrane Detachments with Spontaneous Resolution: Implications for Nonsurgical Treatment. Cornea 28:1160-1163.

Ismail, Z., Fitt, A. D., and Please, C. P. (2013). A fluid mechanical explanation of the spontaneous reattachment of a previously detached Desecement membrance. Mathematical Medicine and Biology 30:339-355.

Jafari, R., and Okutucu-Özyurt, T. (2016). 3D numerical modeling of boiling in a microchannel by arbitrary Lagrangian-Eulerian (ALE) method. Applied Mathematics and Computation 272:593-603.

Jaluria, Y. (1980). Natural Convection Heat and Mass Transfer. London: Pergamon Press.

Karimi, A., Razaghi, R., Navidbakhsh, M., Sera, T., and Kudo, S. (2016a). Computing the influences of different Intraocular Pressures on the human eye components using computational fluid-structure interaction model. Technology and Health Care 1:1-13.

Karimi, A., Razaghi, R., Navidbakhsh, M., Sera, T., and Kudo, S. (2016b). Computing the stresses and deformations of the human eye components due to a high explosive detonation using fluid-structure interaction model. Injury 47:1041-1050.

Karimi, A., Razaghi, R., Navidbakhsh, M., Sera, T., and Kudo, S. (2016c). Quantifying the injury of the human eye components due to tennis ball impact using a computational fluid-structure interaction model. Sports Engineering 19:105-115.

Kjellgren, P., and Hyva Èrinen, J. (1998). An Arbitrary Lagrangian-Eulerian finite element method. Computational Mechanics 21:81-90.

Kuhl, E., Hulsho, S., and de Borst, R. (2003). An arbitrary Lagrangian Eulerian finite-element approach for fluid-structure interaction phenomena. International Journal For Numerical Methods In Engineering 57:117-142.

Kundu, P. K., Cohen, I. M., and Dowling, D. R. (2012). Fluid Mechanics. USA: Academic Press.

Lewis, R. W., Nithiarasu, P., and Seetharamu, K. (2008). Fundamentals of the Finite Element Method for Heat and Fluid Flow: Wiley.

Lo, D. C., and Young, D. L. (2004). Arbitrary Lagrangian-Eulerian finite element analysis of free surface flow using a velocity-vorticity formulation. Journal of Computational Physics 195:175-201.

Marcon, A. S., Rapuano, C. J., Jones, M. R., Laibson, P. R., and Cohen, E. J. (2002). Descemet's membrane detachment after cataract surgery: management and outcome. Ophthalmology 109 (12):2325-2330.

Mulhern, M., Barry, P., and Condon, P. (1996). A case of Descemet's membrane detachment during phacoemulsification surgery. $\mathrm{Br} J$ Ophthalmol. 80 (2):185-186.

Nouri, M., Pineda, R. J., and Azar, D. (2002). Descemet membrane tear after cataract surgery. Seminars in Ophthalmology 17:115-119.

Potter, J., and Zalatimo, N. (2005). Descemet's membrane detachment after cataract extraction. Optometry (St. Louis, Mo.) 76 (12):720-724.

Reddy, J. N., and Gartling, D. K. (2010). The Finite Element Method in Heat Transfer and Fluid Dynamics, Third Edition. USA: Taylor \& Francis.

Sevillano, C., Viso, E., and Millan-Rodriguez, A. C. (2008). Descemet's membrane detachment as a complication of cataract surgery. Archivos de la Sociedad Espaola de Oftalmologa. 83:549-552.

Ünlü, K., and Aksünger, A. (2000). Descemet membrane detachment after viscocanalostomy. American Journal of Ophthalmology 130 (6):833-834.

Ventsel, S., and Krauthammer, T. (2001). Thin Plate and Shells: Theory, Analysis and Application. New York: CRC Press.

Zienkiewicz, O. C., Taylor, R. L., and Nithiarasu, P. (2013). The Finite Element Method for Fluid Dynamics. USA: Elsevier Science. 